\title{
ON NEVANLINNA'S PROXIMITY FUNCTION
}

\author{
SAKARI TOPPILA
}

\section{On the growth of and $T(r, f)$ and $T\left(r, f^{\prime}\right)$}

Let $f$ be a transcendental meromorphic function in the plane. We denote by $T=T(r, f)$ and $T^{\prime}=T\left(r, f^{\prime}\right)$ the characteristic functions of $f$ and $f^{\prime}$. Nevanlinna $[4$, p. 104 , and 5, p. 236$]$ conjectured that

$$
1+o(1) \leqq \frac{T^{\prime}}{T} \leqq 2+o(1)
$$

as $r \rightarrow \infty$ outside an exceptional set $E$ of values of $r$. This conjecture holds in the following form.

Theorem 1. Let $f$ be a transcendental meromorphic function and let $\varphi(r)$ be any positive and increasing function of $r$ such that $\varphi(r) \rightarrow \infty$ as $r \rightarrow \infty$. Then

$$
\begin{aligned}
& \limsup _{r \rightarrow \infty} \frac{T\left(r, f^{\prime}\right)}{T(r+1 / r, f)} \leqq 2, \\
& \liminf _{r \rightarrow \infty} \frac{T\left(r \varphi(r), f^{\prime}\right)}{T(r, f)} \geqq \frac{1}{2},
\end{aligned}
$$

and

$$
\limsup _{r \rightarrow \infty} \frac{T\left(K r, f^{\prime}\right)}{T(r, f)} \geqq 1
$$

for some $K \geqq 1$, and if $f$ is an entire transcendental function, then

$$
\limsup _{r \rightarrow \infty} \frac{T\left(r, f^{\prime}\right)}{T(r+1 / r, f)} \leqq 1
$$

and

$$
\liminf _{r \rightarrow \infty} \frac{T\left(r \varphi(r), f^{\prime}\right)}{T(r, f)} \geqq 1 .
$$

The inequalities (1) and (4) follow from Lemma 1 of Nevanlinna [5, p. 244], (2) and (5) follow from Lemma 1 of Hayman [3, p. 99], and (3) is a consequence of the following result of [7]. 
Theorem 2. Let $f$ be a transcendental meromorphic function of lower order zero. Then

$$
\limsup _{r \rightarrow \infty} \frac{T\left(r, f^{\prime}\right)}{T(r, f)} \geqq 1
$$

and there exists a sequence $r_{n}, r_{n} \rightarrow \infty$ as $n \rightarrow \infty$, such that

$$
m\left(r_{n}, f\right)=m\left(r_{n}, f^{\prime}\right)+o\left(T\left(r_{n}, f\right)\right) \text { as } n \rightarrow \infty .
$$

Theorem 2 shows that we may take $K=1$ in (3) for functions of order zero. If the order of $f$ is infinite, it is not difficult to see that (3) holds for all $K>1$. In the other direction, we have

Theorem 3. Given any $\varepsilon, 0<\varepsilon<\infty$, there exist a meromorphic function $f$ of order $\varepsilon$ and $K>1$ such that

and that for some $\delta>0$,

$$
\limsup _{r \rightarrow \infty} \frac{T\left(K r, f^{\prime}\right)}{T(r, f)}<1
$$

$$
m(r, f) \geqq m\left(r, f^{\prime}\right)+\delta T(r, f)
$$

for all large values of $r$.

Proof. Such a function $f$ is constructed in the proof of Theorem 2 of [7].

If $f$ is an entire function, the following a little stronger result than (3) holds (unpublished).

Theorem 4. There exists an absolute constant $Q>1$ such that

$$
\limsup _{r \rightarrow \infty} \frac{T\left(Q r, f^{\prime}\right)}{T(r, f)} \geqq 1
$$

for any transcendental entire function $f$.

It is not possible to take $Q=1$ in Theorem 4, for in [6] an entire function $f$ of order one is constructed such that $f$ satisfies (8) for some $K>1$.

The following theorem shows that the constant $1 / 2$ in (2) cannot be replaced by a larger one, and that (7) need not hold for all large values of $r$, not even for slowly growing functions.

Theorem 5. Let $\varphi(r)$ be as in Theorem 1. There exists a transcendental meromorphic function $f$ satisfying

$$
T(r, f)=O(\varphi(r) \log r) \quad \text { as } \quad r \rightarrow \infty
$$

such that for some sequences $r_{n}, r_{n} \rightarrow \infty$ as $n \rightarrow \infty$, and $K_{n}, K_{n} \rightarrow \infty$ as $n \rightarrow \infty$,

$$
\begin{gathered}
\lim _{n \rightarrow \infty} \frac{T\left(K_{n} r_{n}, f^{\prime}\right)}{T\left(r_{n}, f\right)}=\frac{1}{2}, \\
m\left(r_{n}, f^{\prime}\right)=0 \text { for any } n,
\end{gathered}
$$


and

$$
m\left(r_{n}, f\right)=\left(\frac{1}{2}+o(1)\right) T\left(r_{n}, f\right) \text { as } n \rightarrow \infty .
$$

Proof. Such a function $f$ is constructed in the proof of Theorem 3 of [7].

For slowly growing functions, Hayman [3] proved the following result stronger than Theorem 1.

Theorem 6. Suppose that $f$ is meromorphic in the plane and not a linear polynomial, further that

$$
T(r, f)=O\left((\log r)^{2}\right) \text { as } r \rightarrow \infty .
$$

Then

$$
\frac{1}{2} \leqq \liminf _{r \rightarrow \infty} \frac{T\left(r, f^{\prime \prime}\right)}{T(r, f)} \leqq \limsup _{r \rightarrow \infty} \frac{T\left(r, f^{\prime}\right)}{T(r, f)} \leqq 2
$$

If, further, $f$ is a transcendental integral function, then

$$
\lim _{r \rightarrow \infty} \frac{T\left(r, f^{\prime}\right)}{T(r, f)}=1 .
$$

In [7] it is proved that the growth condition (15) in Theorem 6 can be replaced by the smoothness condition

$$
\lim _{r \rightarrow \infty} \frac{T(2 r, f)}{T(r, f)}=1 .
$$

If $f$ satisfies (15), then it satisfies (18), too. The following result of Hayman [3] shows that the conditions (15) and (18) are essentially the best possible for Theorem 6.

Theorem 7. Let $\varphi(r)$ be as in Theorem 1. There exists an integral function $f$ such that

$$
T(r, f)=O\left(\varphi(r)(\log r)^{2}\right) \quad \text { as } \quad r \rightarrow \infty
$$

and

$$
\frac{T\left(r, f^{\prime}\right)}{T(r, f)} \rightarrow 0 \text { as } \quad r \rightarrow \infty
$$

through a set of values $E$ having infinite logarithmic measure.

If $f$ has finite order, then

$$
m\left(r, f^{\prime}\right) \leqq m(r, f)+O(\log r) \quad \text { as } \quad r \rightarrow \infty .
$$

This together with the following result of [7] describes the connection between $m(r, f)$ and $m\left(r, f^{\prime}\right)$ for all values of $r$.

Theorem 8. If $f$ is a transcendental meromorphic function satisfying (18), then

$$
m(r, f) \leqq N(r, f)+m\left(r, f^{\prime}\right)+o(T(r, f)) \text { as } r \rightarrow \infty .
$$


Theorem 5 shows that (22) is sharp, and from Theorem 7 we conclude that (15) and (18) are essentially the best possible conditions under which Theorem 8 holds.

Remark. The conditions (3), (6), (7) and (22) do not hold for polynomials. The function $f(z)=z+1 / z$ does not satisfy (7). For rational functions other than polynomials, the conditions (6) and (22) hold.

\section{On the deficiencies of $f$ and $f^{\prime}$}

From the proof of the second main theorem of Nevanlinna [5, pp. 238-247] we get

Theorem 9. Suppose that $f$ is meromorphic in the plane and not a linear polynomial. If the order of $f$ is finite, then

and for any finite $a$

$$
\begin{aligned}
& \delta\left(\infty, f^{\prime}\right) \leqq \delta(\infty, f), \\
& \Delta\left(\infty, f^{\prime}\right) \leqq \Delta(\infty, f), \\
& \sum_{a \neq \infty} \delta(a, f) \leqq 2 \delta\left(0, f^{\prime}\right),
\end{aligned}
$$

If $f$ has infinite or finite order, then

$$
\delta\left(\infty, f^{\prime}\right) \leqq \Delta(\infty, f)
$$

and

$$
\sum_{a \neq \infty} \delta(a, f) \leqq 2 \Delta\left(0, f^{\prime}\right) .
$$

In the other direction, the following theorem is proved in [8]. Then

Theorem 10. Let $f$ be a transcendental meromorphic function satisfying (18).

$$
\begin{aligned}
& \delta\left(\infty, f^{\prime}\right) \geqq 2 \delta(\infty, f)-1, \\
& \Delta\left(\infty, f^{\prime}\right) \geqq \frac{\Delta(\infty, f)}{2-\Delta(\infty f)},
\end{aligned}
$$

with equality in (30) if $f$ has only simple poles, and, furthermore, there exists a finite value a such that

$$
\delta(a, f) \geqq \delta\left(0, f^{\prime}\right) .
$$


From Theorem 2 we get

Theorem 11. If $f$ is a transcendental meromorphic function of order zero, then

$$
\Delta\left(\infty, f^{\prime}\right) \geqq \frac{\delta(\infty, f)}{2-\delta(\infty, f)} .
$$

The condition (29) holds for those rational functions which are not linear polynomials. If we take $f(z)=z+1 / z$, then

and

$$
\Delta(\infty, f)=\delta(\infty, f)=1 / 2
$$

$$
\Delta\left(\infty, f^{\prime}\right)=\delta\left(\infty, f^{\prime}\right)=0 .
$$

For this rational function $f$ the conditions (30) and (32) do not hold. The function

$$
f(z)=\frac{z^{2}+1}{z^{3}}
$$

does not satisfy (31). Modifying a little the function $f$ constructed in [6] we get a meromorphic function of order one which does not satisfy (32). The following result of [8] shows that (29) is sharp.

Theorem 12. Let $\varphi(r)$ be as in Theorem 1. For any $\delta, 1 / 2 \leqq \delta<1$, there exists a transcendental meromorphic function $f$ satisfying (11) such that $\delta(\infty, f)=\delta$ and $\delta\left(\infty, f^{\prime}\right)=2 \delta-1$.

Especially, if $\delta=1 / 2$, then we have $\delta(\infty, f)=1 / 2$ and $\delta\left(\infty, f^{\prime}\right)=0$ in Theorem 12. The following theorem of [8] shows that the conditions (15) and (18) are essentially the best possible for Theorem 10 .

Theorem 13. Let $\varphi(r)$ be as in Theorem 1. There exist transcendental meromorphic functions $f, g$ and $h$ satisfying (19) such that

and

$$
\begin{array}{lll}
\delta\left(\infty, f^{\prime}\right)=0 & \text { but } & \delta(\infty, f)=1, \\
\Delta\left(\infty, g^{\prime}\right)=0 & \text { but } & \Delta(\infty, g)=1,
\end{array}
$$

for all values $a$.

$$
\delta\left(0, h^{\prime}\right)>0 \quad \text { but } \quad \delta(a, h)=0
$$

Let $g$ be an entire transcendental function with simple zeros satisfying (15). Then we see from Theorem 6 that

$$
T\left(r, g^{\prime}\right)=(1+o(1)) T(r, g) \text { as } r \rightarrow \infty .
$$

Using Theorem 1 of Hayman [2] we conclude that

$$
\begin{aligned}
N\left(r, 0, g^{\prime}\right) & =\left(1+o(1) T\left(r, g^{\prime}\right)\right. \\
& =(1+o(1)) T(r, g) \\
& =(1+o(1)) N(r, 0, g) \text { as } r \rightarrow \infty,
\end{aligned}
$$


and that the function $f=1 / g$ satisfies

$$
T\left(r, f^{\prime}\right)=(2+o(1)) N(r, 0, g) \text { as } r \rightarrow \infty .
$$

Since $N\left(r, 0, f^{\prime}\right)=N\left(r, 0, g^{\prime}\right)$, we conclude that $\delta\left(0, f^{\prime}\right)=\Delta\left(0, f^{\prime}\right)=1 / 2$. Clearly

$$
\delta(0, f)=\Delta(0, f)=1 .
$$

This example shows that the constant 2 in the inequalities (25), (26) and (28) cannot be replaced by a smaller one, not even for slowly growing functions.

The conditions (23), (24), (25) and (26) need not hold for functions of infinite order. In fact, there exist meromorphic functions $f, g$ and $h$ of infinite order such that

and

$$
\begin{array}{ccll}
\delta(\infty, f)=0 & \text { but } & \delta\left(\infty, f^{\prime}\right)=1, \\
\Delta(\infty, g)=0 & \text { but } & \Delta\left(\infty, g^{\prime}\right)=1, \\
\delta(0, g)=1 & \text { but } & \delta\left(0, g^{\prime}\right)=0,
\end{array}
$$

$$
\Delta(0, h)=1 \text { but } \Delta\left(0, h^{\prime}\right)=0 .
$$

For a proof, we refer to [8] and [9].

\section{References}

[1] Anderson, J. M.: Asymptotic values of meromorphic functions of smooth growth. - Glasgow Math. J. 20, 1979, 155-162.

[2] Hayman, W. K.: Slowly growing integral and subharmonic functions. - Comment. Math. Helv. $34,1960,75-84$.

[3] Hayman, W. K.: On the characteristic of functions meromorphic in the plane and of their integrals. - Proc. London Math. Soc. (3) 14 A, 1965, 93-128.

[4] Nevanlinna, R.: Le théorème de Picard-Borel et la théorie des fonctions méromorphes. Gauthier-Villars et $C^{\text {ie }}$, Paris, 1929.

[5] Nevanlinna, R.: Analytic functions. - Die Grundlehren der mathematischen Wissenschaften 162, Springer-Verlag, Berlin-Heidelberg-New York, 1970.

[6] ToppILA, S.: On Nevanlinna's characteritic function of entire functions and their derivatives. Ann. Acad. Sci. Fenn. Ser. A I Math. 3, 1977, 131-134.

[7] ToppILA, S.: On the characteristic of meromorphic functions and their derivatives. - J. London Math. Soc. (2) (to appear).

[8] Toppila, S.: On the deficiencies of a meromorphic function and of its derivative. - J. London Math. Soc. (2) (to appear).

[9] Toppila, S.: An introduction to Nevanlinna theory. - To appear.

University of Helsinki

Department of Mathematics

SF-00100 Helsinki 10

Finland

Received 5 March 1981 